\title{
PROPERTIES OF OLIGOPOLISTIC MARKET EQUILIBRIA IN LINEARIZED DC POWER NETWORKS WITH ARBITRAGE AND SUPPLY FUNCTION CONJECTURES
}

\author{
Jong-Shi Pang \\ Department of Mathematical Sciences \\ The Johns Hopkins University \\ Baltimore, Maryland 21218-2682, U.S.A. \\ jsp@vicp1.mts.jhu.edu
}

Benjamin F. Hobbs

Department of Geography and Environmental Engineering

The Johns Hopkins University

Baltimore, Maryland 21218-2682, U.S.A.

bhobbs@jhu.edu

\author{
Christopher J. Day \\ Enron Europe Limited \\ 40 Grosvenor Place \\ London SW1X YEN, United Kingdom \\ christopher.j.day@enron.com
}

\begin{abstract}
We present mathematical models for a power market on a linearized DC network with affine demand. The models represent the conjecture that each power generating company may hold regarding how rival firms will change their outputs if prices change. The classic Cournot model is a special case of this conjecture. The models differ in how arbitrage is handled, and their formulations give rise to nonlinear mixed complementarity problems. In the Stackelberg version, the generators anticipate how arbitrage would affect prices at different locations, and therefore treat the arbitrage amounts as decision variables in their profit maximization problems. In the other version, arbitrage is exogenous to the
\end{abstract}

The original version of this chapter was revised: The copyright line was incorrect. This has been corrected. The Erratum to this chapter is available at DOI: 10.1007/978-0-387-35699-0_19 
firms. We show that solutions to the latter model are also solutions to the Stackelberg model. We also demonstrate existence and uniqueness properties for the exogenous arbitrage model.

\section{Introduction}

In restructured power markets, electric power generators have been privatized or freed of regulatory constraints on prices. The intent of restructuring is to provide incentives for innovation and more efficient production and consumption of electricity [5]. However, because of market failures, these benefits may not be fully realized. A market failure that has been of particular concern to regulators and the public is market power [12]. Market power is defined as the ability of a market participant to unilaterally alter prices in its own favor, and to sustain those price changes. Transmission capacity limits that restrict power imports and exports are an important source of market power for generating companies, as they allow firms within an isolated region to raise prices above competitive levels [2].

The potential for market power to be exercised within a given power system can be studied through laboratory experiments, empirical analysis, and modeling. There are many models of strategic interaction in transmission constrained systems (for reviews, see [7] or [8]). Models can be used to unveil unanticipated ways in which market power might be exercised on networks, to identify locations where prices can be manipulated, to assess the effects of adding transmission capacity upon prices, and to examine the competitive effects of company mergers or divestments. The most common oligopolistic modeling frameworks employed in power market analyses are based on the ideas of Cournot games and Supply Function Equilibria (SFE), defined below.

The purpose of this paper is to analyze the existence and uniqueness properties of solutions of a new model of oligopolistic power generators. The model represents the power network using a linearized "DC" load flow model [13], and includes a flexible representation of interactions of competing generating firms. We term this representation the "conjectured supply functions" (CSFs) approach. A CSF is a function representing the beliefs of a firm concerning how total supply from rival firms will react to price. Two versions of a linear CSF have been proposed: one in which the slope of conjectured supply response is constant and the intercept is to be solved for, and another in which the intercept is given but the slope is to be determined. The former CSP yields a linear mixed complementarity problem (MCP) for the market equilibrium, while the latter gives a nonlinear MCP. 
The CSF model can be viewed as a generalization of the Cournot models of [7] and [14] in that the amount that rival firms are anticipated to adjust prices in response to a price change is not restricted to zero (the Cournot assumption). Instead, each generating company is allowed to conjecture that rival firms will react to price increases or decreases. By making different assumptions about the assumed supply response, different degrees of competitive intensity can be modeled, ranging from pure competition (infinitely large positive response by rivals to price increases) to oligopolistic Cournot competition (no response). Positive sloped CSFs represent a competitive intensity between the Cournot and pure competition extremes. A detailed justification of the CSF approach to modeling competition on transmission networks is given in [4], along with an application to the United Kingdom power system.

It should be noted that the CSF modeling approach is distinct from the widely used supply function equilibrium (SFE) approach to market modeling $[1,9]$. The SFE is a Nash game in bid functions, in which suppliers provide a function to a central auctioneer that relates their willingness to supply to the price. The SFE approach also yields prices intermediate between the pure competition and Cournot extremes, but is plagued by computational challenges along with problems of nonuniqueness and, in some cases, nonexistence of solutions [2]. The fundamental difference between the SFE and CSF approaches is that the anticipated supply response of competitors is endogenous in SFE models and is consistent with the competitor's actual bid function, while in the CSF approach, the conjectured supply response of competing firms is instead based on an assumed parameter (slope or intercept). It is this difference that allows SFEs to be formulated as mixed complementarity problems that are relatively easy to solve and yield solutions whose existence and uniqueness properties can be demonstrated.

Questions concerning the existence and uniqueness of equilibrium solutions to market models are important for two reasons. First, public policy is in part based on policy analyses using market models; if unique solutions cannot be assured, then the question arises as to whether the conclusions of an analysis depend on which of several possible solutions is selected. Second, if a solution exists and is unique, then computational procedures do not need to check for multiple solutions, and are therefore simpler. This paper focuses on the existence and uniqueness properties of the solution of the nonlinear MCP (fixed intercept model), as those properties for the linear MCP (fixed slope) are readily established using the results of [11].

The paper begins by defining notation and the profit maximizing problems that are common to all the models presented in this paper 
(Section 2). Those common problems include the profit maximization problems for the independent system operator (ISO) who allocates scarce transmission capacity, and the arbitrager who eliminates any noncostbased price differences among nodes in the network. Consumers are represented by downward sloping demand curves. The various models introduced in the paper differ in terms of their representation of the profit maximization problem for the oligopolistic power producer. The first model, Model I, is introduced in Section 3. There, the power producer makes production and sales decisions recognizing that demand responds to price, that rival producers will react to price changes (according to the assumed CSF), and that noncost-based price differences will be arbitraged away. Inclusion of arbitrage means that the arbitrager's equilibrium conditions are introduced as constraints in the producer's constraint set. After introducing the producer profit maximization problem, we obtain the nonlinear MCP that represents the market equilibrium. Section 4 presents Model II which differs from Model I in that the arbitrager's equilibrium conditions are kept outside of the producer's problem, resulting in a model which can be analyzed more fully than Model I. In Section 5, relevant theory of monotone linear complementarity problems is introduced which will be the basis for the demonstrations of the model properties. This theory is used in Section 6 to establish the existence of solutions to Models I and II, the conditions under which solutions to Model II exist and certain of the variables (prices, total generation, sales, and profits) are unique.

\section{The ISO and Arbitrage Models}

In this and the next two sections, we present the mixed NCP formulations of the market equilibrium with conjectured supply functions. The resulting models become the respective linear complementarity models considered in [11] when the intercepts tend to minus infinity. In what follows, we present the NCP models, establish the existence of solutions and analyze their properties.

\section{$2.1 \quad$ Notation}

Before presenting the mathematical formulations for the models, we summarize the notation.

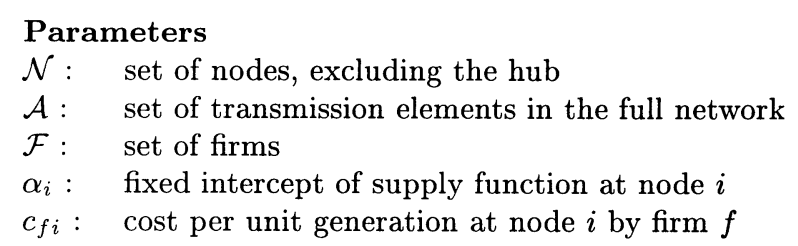


$P_{i}^{0}: \quad$ price intercept of supply function at node $i$

$Q_{i}^{0}: \quad$ quantity intercept of supply function at node $i$

$T_{k}^{+}$: $\quad$ capacity on transmission element $k$

$T_{k}^{-}: \quad$ capacity in the reverse direction of transmission element $k$

$\mathrm{CAP}_{f i}$ : $\quad$ production capacity at node $i$ for firm $f$

$\mathrm{PDF}_{i k}$ : $\quad$ power distribution factor for node $i$ on element $k$, describing the megawatt (MW) increase in flow resulting from $1 \mathrm{MW}$ of power injection at $i$ and $1 \mathrm{MW}$ of withdrawal at a hub node.

\section{Variables}

$s_{f i}: \quad$ amount of sales at node $i$ by firm $f$

$g_{f i}: \quad$ generation at node $i$ by firm $f$

$p_{f i}: \quad$ price at node $i$ anticipated by firm $f$

$y_{i}$ : amount of transmission service from hub $H$ to node $i$

$w_{i}: \quad$ transmission price from hub $H$ to node $i$

$p_{f}: \quad$ price at the hub node, anticipated by firm $f$

$a_{f i}$ : amount that arbitragers sell at node $i$, anticipated by firm $f$

$\lambda_{k}^{ \pm}$: dual variables of transmission capacity constraints in ISO's problem

$\gamma_{f i}$ : dual variable of production capacity constraint in firm $f$ 's problem

$\varphi_{f}:$ dual variable of balance equation between supply and generation in firm $f$ 's problem

$\pi_{i}: \quad$ market price at node $i$

\section{Vectors \& Matrices}

1: $\quad$ vector of ones of appropriate size

I: $\quad$ identity matrix of appropriate order

$\boldsymbol{E}: \quad$ square matrix of ones of appropriate order

П: $\quad|\mathcal{N}| \times|\mathcal{A}|$ matrix of $\mathrm{PDF}_{i k}, i \in \mathcal{N}$ and $k \in \mathcal{A}$

$s: \quad(|\mathcal{N}| \times|\mathcal{F}|)$-vector of $s_{f i}, i \in \mathcal{N}$ and $f \in \mathcal{F}$

g: $\quad(|\mathcal{N}| \times|\mathcal{F}|)$-vector of $g_{f i}, i \in \mathcal{N}$ and $f \in \mathcal{F}$

$\pi: \quad|\mathcal{N}|$-vector of equilibrium prices $\pi_{i}, i \in \mathcal{N}$

$\gamma: \quad(|\mathcal{N}| \times|\mathcal{F}|)$-vector of $\gamma_{f i}, i \in \mathcal{N}$ and $f \in \mathcal{F}$

$\lambda^{ \pm}: \quad|\mathcal{A}|$-vectors of $\lambda_{k}^{ \pm}, k \in \mathcal{A}$

$c: \quad|\mathcal{N}| \times|\mathcal{F}|)$-vector of $c_{f i}, i \in \mathcal{N}$ and $f \in \mathcal{F}$

CAP: $\quad(|\mathcal{N}| \times|\mathcal{F}|)$-vector of $\mathrm{CAP}_{f i}, i \in \mathcal{N}$ and $f \in \mathcal{F}$.

The components of the vectors $\boldsymbol{s}, \boldsymbol{g}, c$, and CAP are grouped by firms; that is

$$
s \equiv\left(s_{1}, \ldots, s_{|\mathcal{F}|}\right)^{T}
$$

where each $s_{f}$ is the $|\mathcal{N}|$-vector with components $s_{f i}, i \in \mathcal{N}$. The other three vectors $\boldsymbol{g}, \boldsymbol{c}$, and CAP are similarly arranged. Except for the supply intercepts and some power distribution factors, all parameters of the models are positive. 


\subsection{The ISO's problem}

The ISO's problem is the following linear program (LP). Given the transmission prices $w_{i}, i \in \mathcal{N}$, compute $y_{i}, i \in \mathcal{N}$ in order to

$$
\begin{aligned}
\operatorname{maximize} & \sum_{i \in \mathcal{N}} w_{i} y_{i} \\
\text { subject to } & \sum_{i \in \mathcal{N}} y_{i}=0, \\
& \sum_{i \in \mathcal{N}} \mathrm{PDF}_{i k} y_{i} \leq T_{k}^{+}, \quad \forall k \in \mathcal{A}, \quad\left(\lambda_{k}^{+}\right) \\
& \sum_{i \in \mathcal{N}} \mathrm{PDF}_{i k} y_{i} \geq-T_{k}^{-}, \quad \forall k \in \mathcal{A}, \quad\left(\lambda_{k}^{-}\right),
\end{aligned}
$$

where we write the dual variables in parentheses next to the corresponding constraints. Note that the variables $y_{i}$ are unrestricted in sign. A positive (negative) $y_{i}$ means that there is a net flow into (out of) node $i$. It is trivial to note that $y=0$ is always a feasible solution to the above LP, because the $T_{k}^{ \pm}$are positive scalars. The optimality conditions of the LP can be written as a mixed LCP in the variables $y_{i}$ for $i \in \mathcal{N}, \lambda_{k}^{ \pm}$ for $k \in \mathcal{A}$ and $\eta$, parameterized by the transmission fees $w_{i}, i \in \mathcal{N}$ :

$$
\begin{aligned}
& 0 \leq \lambda_{k}^{-} \perp T_{k}^{-}+\sum_{i \in \mathcal{N}} \operatorname{PDF}_{i k} y_{i} \geq 0, \quad k \in \mathcal{A}, \\
& 0 \leq \lambda_{k}^{+} \perp T_{k}^{+}-\sum_{i \in \mathcal{N}} \operatorname{PDF}_{i k} y_{i} \geq 0, \quad k \in \mathcal{A}, \\
& 0=\sum_{i \in \mathcal{N}} y_{i}, \\
& 0=w_{i}+\sum_{k \in \mathcal{A}} \operatorname{PDF}_{i k}\left(\lambda_{k}^{-}-\lambda_{k}^{+}\right)+\eta, \quad i \in \mathcal{N} .
\end{aligned}
$$

\subsection{The arbitrager's problem}

The arbitrager maximizes its profit by buying and selling power in the market, given the prices at the nodes in the network. With $a_{i}$ denoting the arbitrage amount sold at node $i$, the arbitrager's profit maximization problem is very simple: for fixed prices $p_{i}$ and costs $w_{i}$, compute $a_{i}$, $i \in \mathcal{N}$ in order to

$$
\begin{array}{ll}
\operatorname{maximize} & \sum_{i \in \mathcal{N}}\left(p_{i}-w_{i}\right) a_{i} \\
\text { subject to } & \sum_{i \in \mathcal{N}} a_{i}=0, ;
\end{array}
$$


the transmission fee at node $i$ is included in the objective function because the arbitrager must also pay this cost. The arbitrage amounts are measured as the net sales at a node; thus the sum of all the arbitrage amounts must equal to zero. Note that $a_{i}$ is unrestricted in sign. A positive $a_{i}$ represents the amount sold by the arbitrager at node $i$; in this case, the arbitrager is receiving $p_{i}$ for each unit sold but is paying $w_{i}$ for the transmission. If $a_{i}$ is negative, then $\left|a_{i}\right|$ is the quantity that the arbitrager bought from node $i$; in this case, the arbitrager is paying $p_{i}$ per unit and paying $-w_{i}$ per unit to ship out of $i$.

The problem (3) is trivially solvable. In particular, this problem is equivalent to the two equations:

$$
\begin{aligned}
p_{i}-w_{i}-p_{H} & =0, \quad \forall i \in \mathcal{N} \\
\sum_{i \in \mathcal{N}} a_{i} & =0 .
\end{aligned}
$$

In turn, the first equation implies

$$
p_{i}-p_{j}=w_{i}-w_{j}, \quad \forall i, j \in \mathcal{N},
$$

which says that the difference in prices at two distinct nodes is exactly the difference between the transmission fees at those two nodes.

\section{Model I}

In this model, each firm that produces power anticipates the arbitrage amounts by including the variables $a_{f i}$ and a supply function conjecture with fixed intercept in its profit maximization problem. The constraints that these variables satisfy are basically (4), where $p_{i}$ is determined by the price function:

$$
p_{f i} \equiv P_{i}^{0}-\frac{P_{i}^{0}}{Q_{i}^{0}}\left(\sum_{t \in \mathcal{F}} s_{t i}+a_{f i}\right) .
$$

(Note the addition of the subscript $i$ in $p_{f i}$ as this is now the price at $i$ anticipated by $f$.) The supply function conjecture is expressed by the equation

$$
s_{-f i} \equiv \sum_{t \neq f} s_{t i}=\frac{p_{f i}-\alpha_{i}}{\pi_{i}-\alpha_{i}} s_{-f i}^{*} .
$$

Note that $\pi_{i}$ is a base price at which $s_{-f i}=s_{-f i}^{*}$ and is exogenous to the firms. Substituting $s_{-f i}$ into the former equation and simplifying, we obtain

$$
p_{f i}=\left(Q_{i}^{0}-s_{f i}-a_{f i}+\frac{\alpha_{i}}{\pi_{i}-\alpha_{i}} s_{-f i}^{*}\right) /\left(\frac{Q_{i}^{0}}{P_{i}^{0}}+\frac{s_{-f i}^{*}}{\pi_{i}-\alpha_{i}}\right) .
$$


Letting $p_{f}$ be the firm's anticipated price at the hub, firm $f$ 's problem is: with $s_{-f i}^{*}, \pi_{i}$, and $w_{i}, i \in \mathcal{N}$ fixed, find $s_{f i}, g_{f i}, a_{f i}, p_{f i}$ for $i \in \mathcal{N}$, and $p_{f}$ in order to

$$
\begin{array}{ll}
\operatorname{maximize} & \sum_{i \in \mathcal{N}}\left(p_{f i}-w_{i}\right) s_{f i}-\sum_{i \in \mathcal{N}}\left(c_{f i}-w_{i}\right) g_{f i} \\
\text { subject to } & g_{f i} \leq \mathrm{CAP}_{f i}, \quad \forall i \in \mathcal{N} \\
& \sum_{i \in \mathcal{N}}\left(s_{f i}-g_{f i}\right)=0, \\
& p_{f i}=\frac{Q_{i}^{0}-s_{f i}-a_{f i}+\frac{\alpha_{i}}{\pi_{i}-\alpha_{i}} s_{-f i}^{*}}{\frac{Q_{i}^{0}}{P_{i}^{0}}+\frac{s_{-f i}^{*}}{\pi_{i}-\alpha_{i}}, \quad \forall i \in \mathcal{N}} \\
& p_{f i}=p_{f}+w_{i}, \quad \forall i \in \mathcal{N} \\
& \sum_{i \in \mathcal{N}} a_{f i}=0, \quad \forall i \in \mathcal{N} . \\
& s_{f i}, g_{f i} \geq 0, \quad \forall i
\end{array}
$$

The three equations

$$
\begin{aligned}
& p_{f i}=\left(Q_{i}^{0}-s_{f i}-a_{f i}+\frac{\alpha_{i}}{\pi_{i}-\alpha_{i}} s_{-f i}^{*}\right) /\left(\frac{Q_{i}^{0}}{P_{i}^{0}}+\frac{s_{-f i}^{*}}{\pi_{i}-\alpha_{i}}\right), \quad \forall i \in \mathcal{N} \\
& p_{f i}=p_{f}+w_{i}, \quad \forall i \in \mathcal{N} \\
& \sum_{i \in \mathcal{N}} a_{f i}=0,
\end{aligned}
$$

uniquely determine $p_{f i}$ and $a_{f i}$ for $i \in \mathcal{N}$ and $p_{f}$ in terms of $s_{t j}, w_{j}$ and $\pi_{j}$ for all $t \in \mathcal{F}$ and $j \in \mathcal{N}$. For the purpose of restating firm $f$ 's maximization problem, it suffices to solve for $p_{f}$, obtaining,

$$
p_{f}=\frac{\sum_{i \in \mathcal{N}} Q_{i}^{0}-\sum_{i \in \mathcal{N}} w_{i}\left(\frac{Q_{i}^{0}}{P_{i}^{0}}+\frac{s_{-f i}^{*}}{\pi_{i}-\alpha_{i}}\right)-\sum_{i \in \mathcal{N}} s_{f i}+\sum_{i \in \mathcal{N}} \frac{\alpha_{i}}{\pi_{i}-\alpha_{i}} s_{-f i}^{*}}{\sum_{i \in \mathcal{N}}\left(\frac{Q_{i}^{0}}{P_{i}^{0}}+\frac{s_{-f i}^{*}}{\pi_{i}-\alpha_{i}}\right) .}
$$


Let $p_{f}(\boldsymbol{s}, \boldsymbol{\pi}, \boldsymbol{w})$ denote the fraction on the right-hand side as a function of the vectors $s, \pi$, and $\boldsymbol{w}$. This function depends on the intercepts $\alpha_{i}$; but since these are parameters of the model, we do not write them as the arguments of $p_{f}$. The function $p_{f}$ also depends on $s_{-f i}^{*}$ and $\pi_{i}$; at equilibrium, the latter variables will be equated with $s_{-f i}$ and $p_{f i}$, respectively. Observe that $p_{f}$ is a linear function of $s_{f}$, with the other arguments fixed.

We can now restate firm $f$ 's problem in the simplified form: with $s_{t i}$ $(t \neq f), \pi_{i}$, and $w_{i}, i \in \mathcal{N}$ fixed, find $s_{f i}$ and $g_{f i}$ for $i \in \mathcal{N}$ in order to

$$
\begin{array}{ll}
\operatorname{maximize} & \sum_{i \in \mathcal{N}} p_{f}(\boldsymbol{s}, \boldsymbol{\pi}, \boldsymbol{w}) s_{f i}-\sum_{i \in \mathcal{N}}\left(c_{f i}-w_{i}\right) g_{f i} \\
\text { subject to } & g_{f i} \leq \mathrm{CAP}_{f i}, \quad \forall i \in \mathcal{N}, \quad\left(\gamma_{f i}\right) \\
& \sum_{i \in \mathcal{N}}\left(s_{f i}-g_{f i}\right)=0, \quad\left(\varphi_{f}\right) \\
& s_{f i}, g_{f i} \geq 0, \quad \forall i \in \mathcal{N} .
\end{array}
$$

The above problem is a quadratic concave maximization problem in the variables $s_{f i}$ and $g_{f i}$ for $i \in \mathcal{N}$, parameterized by $s_{t i}$ for $t \neq f$ and $\pi_{i}$ and $w_{i}$ for $i \in \mathcal{N}$. We can write the optimality conditions for the problem as follows:

$$
\begin{array}{ll}
0 \leq s_{f i} \perp-p_{f}(\boldsymbol{s}, \boldsymbol{\pi}, \boldsymbol{w})+\frac{\sum_{j \in \mathcal{N}} s_{f j}}{\sum_{j \in \mathcal{N}}\left(\frac{Q_{j}^{0}}{P_{j}^{0}}+\frac{s_{-f j}^{*}}{\pi_{j}-\alpha_{j}}\right)}+\varphi_{f} \geq 0, & i \in \mathcal{N}, \\
0 \leq g_{f i} \perp c_{f i}-w_{i}+\gamma_{f i}-\varphi_{f} \geq 0, & i \in \mathcal{N}, \\
0 \leq \gamma_{f i} \perp \mathrm{CAP}_{f i}-g_{f i} \geq 0, & i \in \mathcal{N}, \\
0=\sum_{i \in \mathcal{N}}\left(s_{f i}-g_{f i}\right) . &
\end{array}
$$

To complete the description of the model, we need to relate the ISO's problem to the firms' problems. This is accomplished via the market clearing condition, which is simply a flow balancing equation:

$$
y_{i}=\sum_{t \in \mathcal{F}}\left(s_{t i}-g_{t i}\right)+a_{f i}, \quad \forall(f, i) \in \mathcal{F} \times \mathcal{N} .
$$

In addition, we stipulate that

$$
p_{f}(\boldsymbol{s}, \boldsymbol{\pi}, \boldsymbol{w})+w_{i}=\pi_{i}, \quad \forall(f, i) \in \mathcal{F} \times \mathcal{N}
$$


and $s_{-f i}^{*}=s_{-f i}$ for all $(f, i) \in \mathcal{F} \times \mathcal{N}$. From the definition of $p_{f}(\boldsymbol{s}, \boldsymbol{\pi}, \boldsymbol{w})$, the last two conditions yield

$$
S \equiv \sum_{f \in \mathcal{F}} \sum_{i \in \mathcal{N}} s_{f i}=\sum_{i \in \mathcal{N}} Q_{i}^{0}-\sum_{i \in \mathcal{N}} \pi_{i} \frac{Q_{i}^{0}}{P_{i}^{0}},
$$

which expresses the total sales $S$ of all firms in all markets in terms of the market prices $\pi_{i}$. Substituting (6) into the last equation, we obtain

$$
p_{f}(\boldsymbol{s}, \boldsymbol{\pi}, \boldsymbol{w})=\left(\sum_{i \in \mathcal{N}} Q_{i}^{0}-\sum_{i \in \mathcal{N}} w_{i} \frac{Q_{i}^{0}}{P_{i}^{0}}-S\right) /\left(\sum_{i \in \mathcal{N}} \frac{Q_{i}^{0}}{P_{i}^{0}},\right)
$$

which shows among other things that $p_{f}(\boldsymbol{s}, \boldsymbol{\pi}, \boldsymbol{w})$ is the same for all firms and

$$
p_{f i}=p_{f}(\boldsymbol{s}, \boldsymbol{\pi}, \boldsymbol{w})+w_{i}=\pi_{i}
$$

thus the price $p_{f i}$ is independent of the firms. Substituting the equality $p_{f i}=\pi_{i}$ into the expression

$$
p_{f i}=\left(Q_{i}^{0}-s_{f i}-a_{f i}+\frac{\alpha_{i}}{\pi_{i}-\alpha_{i}} s_{-f i}\right) /\left(\frac{Q_{i}^{0}}{P_{i}^{0}}+\frac{s_{-f i}}{\pi_{i}-\alpha_{i}}\right)
$$

yields

$$
a_{f i}=Q_{i}^{0}-\pi_{i} \frac{Q_{i}^{0}}{P_{i}^{0}}-\sum_{t \in \mathcal{F}} s_{t i}
$$

which shows that the arbitrage amounts anticipated by the firms depend only on the market $i$. Substituting the expression

$$
\begin{aligned}
\pi_{i} & =\left(\sum_{j \in \mathcal{N}} Q_{j}^{0}-\sum_{j \in \mathcal{N}} w_{j} \frac{Q_{j}^{0}}{P_{j}^{0}}-S\right) /\left(\sum_{j \in \mathcal{N}} \frac{Q_{j}^{0}}{P_{j}^{0}}\right)+w_{i} \\
& =\sigma+\sum_{j \in \mathcal{N}}\left(\delta_{i j}-\rho_{i}\right) w_{j}-\omega S
\end{aligned}
$$

into the above expression for $a_{f i}$, we obtain

$$
a_{f i}=R_{i}^{0}-\sum_{t \in \mathcal{F}} s_{t i}+\rho_{i} S-\sum_{j \in \mathcal{N}} \zeta_{i j} w_{j}
$$

where

$$
\begin{aligned}
\omega & \equiv \frac{1}{\sum_{i \in \mathcal{N}} \frac{Q_{i}^{0}}{P_{i}^{0}}}, \quad \sigma \equiv \omega \sum_{i \in \mathcal{N}} Q_{i}^{0}, \\
\rho_{i} \equiv \omega \frac{Q_{i}^{0}}{P_{i}^{0}}, & R_{i}^{0} \equiv Q_{i}^{0}-\frac{Q_{i}^{0}}{P_{i}^{0}} \sigma, \quad i \in \mathcal{N},
\end{aligned}
$$


and with $\delta_{i j}$ denoting the Kronecker delta; i.e., $\delta_{i j}$ is equal to one if $i=j$ and equal to zero if $i \neq j$,

$$
\begin{aligned}
\zeta_{i j} & \equiv \frac{Q_{i}^{0}}{P_{i}^{0}}\left(\delta_{i j}-\rho_{j}\right), \quad i, j \in \mathcal{N} \\
& = \begin{cases}\frac{Q_{i}^{0}}{P_{i}^{0}}\left(1-\omega \frac{Q_{i}^{0}}{P_{i}^{0}}\right) & \text { if } i=j \\
-\omega \frac{Q_{i}^{0}}{P_{i}^{0}} \frac{Q_{j}^{0}}{P_{j}^{0}} & \text { if } i \neq j .\end{cases}
\end{aligned}
$$

Substituting the above expression for $a_{f i}$ into (5), we obtain

$$
y_{i}=R_{i}^{0}-\sum_{t \in \mathcal{F}} g_{t i}+\rho_{i} S-\sum_{j \in \mathcal{N}} \zeta_{i j} w_{j}
$$

Proceeding as in [11], we can show that the resulting Model $\mathrm{I}$ is to compute

$$
\left\{\lambda_{k}^{ \pm}: k \in \mathcal{A}\right\},\left\{s_{f i}, g_{f i}, \gamma_{f i}: i \in \mathcal{N}, f \in \mathcal{F}\right\}, \text { and }\left\{\varphi_{f}^{\prime}: f \in \mathcal{F}\right\}
$$

such that

$$
\begin{aligned}
& 0 \leq \lambda_{k}^{-} \perp q_{k}^{-}+\sum_{\ell \in \mathcal{A}}\left[\sum_{i, j \in \mathcal{N}} \operatorname{PDF}_{i k} \zeta_{i j} \mathrm{PDF}_{j \ell}\right]\left(\lambda_{\ell}^{-}-\lambda_{\ell}^{+}\right)+\left(\sum_{i \in \mathcal{N}} \rho_{i} \mathrm{PDF}_{i k}\right) S-\sum_{i \in \mathcal{N}} \sum_{f \in \mathcal{F}} \mathrm{PDF}_{i k} g_{f i} \geq 0, \quad \forall k \in \mathcal{A}, \\
& 0 \leq \lambda_{k}^{+} \perp q_{k}^{+}+\sum_{\ell \in \mathcal{A}}\left[\sum_{i, j \in \mathcal{N}} \mathrm{PDF}_{i k} \zeta_{i j} \mathrm{PDF}_{j \ell}\right]\left(\lambda_{\ell}^{+}-\lambda_{\ell}^{-}\right)- \\
&\left(\sum_{i \in \mathcal{N}} \rho_{i} \mathrm{PDF}_{i k}\right) S+\sum_{i \in \mathcal{N}} \sum_{f \in \mathcal{F}} \mathrm{PDF}_{i k} g_{f i} \geq 0, \quad \forall k \in \mathcal{A}, \\
& 0 \leq s_{f i} \perp \quad\left.-\sigma+\omega S+\frac{\sum_{j \in \mathcal{N}} s_{f j}}{\sum_{j \in \mathcal{N}}\left(\frac{Q_{j}^{0}}{P_{j}^{0}}+\frac{s_{-f j}}{\pi_{j}-\alpha_{j}}\right)}+\varphi_{f}^{\prime}\right) \\
& \sum_{k \in \mathcal{A}}\left[\sum_{j \in \mathcal{N}} \rho_{j} \mathrm{PDF}_{j k}\right]\left(\lambda_{k}^{+}-\lambda_{k}^{-}\right) \geq 0, \forall(f, i) \in \mathcal{F} \times \mathcal{N},
\end{aligned}
$$




$$
\begin{aligned}
& 0 \leq g_{f i} \perp \quad c_{f i}+\sum_{k \in \mathcal{A}} \operatorname{PDF}_{i k}\left(\lambda_{k}^{-}-\lambda_{k}^{+}\right)+ \\
& \gamma_{i f}-\varphi_{f}^{\prime} \geq 0, \quad \forall(f, i) \in \mathcal{F} \times \mathcal{N}, \\
& 0 \leq \gamma_{f i} \perp \quad \operatorname{CAP}_{f i}-g_{f i} \geq 0, \quad \forall(f, i) \in \mathcal{F} \times \mathcal{N}, \\
& 0=\quad \sum_{i \in \mathcal{N}}\left(s_{f i}-g_{f i}\right), \quad \forall f \in \mathcal{F},
\end{aligned}
$$

where

$$
q_{k}^{ \pm} \equiv T_{k}^{ \pm} \mp \sum_{i \in \mathcal{N}} R_{i}^{0} \mathrm{PDF}_{i k}, \quad \forall k \in \mathcal{A} .
$$

In vector form, we have,

$$
q^{ \pm}=T^{ \pm} \mp \Pi^{T} R^{0}=T^{ \pm} \mp \Pi^{T} \Xi P^{0} .
$$

We observe that

$$
\begin{aligned}
\pi_{i} & =\sigma-\omega S+\sum_{j \in \mathcal{N}} \sum_{k \in \mathcal{A}}\left(\delta_{i j}-\rho_{j}\right) \operatorname{PDF}_{j k}\left(\lambda_{k}^{+}-\lambda_{k}^{-}\right) \\
& =\sigma-\omega S+\frac{P_{i}^{0}}{Q_{i}^{0}} \sum_{j \in \mathcal{N}} \sum_{k \in \mathcal{A}} \zeta_{i j} \operatorname{PDF}_{j k}\left(\lambda_{k}^{+}-\lambda_{k}^{-}\right),
\end{aligned}
$$

which expresses the regional prices $\pi_{i}$ in terms of the total sales $S$ and the dual variables of the transmission capacity constraints. Subsequently, we show that $\pi_{i}$ is uniquely determined by the total sales only.

Let $h_{\mathrm{I}}: \Re^{|\mathcal{F}| \times|\mathcal{N}|+2|\mathcal{A}|} \rightarrow \Re^{|\mathcal{F}| \times|\mathcal{N}|}$ be defined by

$$
h_{\mathrm{I}, f}\left(s, \lambda^{ \pm}\right) \equiv \frac{\sum_{j \in \mathcal{N}} s_{f j}}{\sum_{j \in \mathcal{N}}\left(\frac{Q_{j}^{0}}{P_{j}^{0}}+\frac{s_{-f j}}{\pi_{j}-\alpha_{j}}\right)} \mathbf{1}_{|\mathcal{N}|}, \quad \forall f \in \mathcal{F},
$$

where $\pi_{i}$ is given by (9). We can now write Model I in vector-matrix form. First we assemble the variables of the system in two vectors:

$$
\boldsymbol{x} \equiv\left(\begin{array}{c}
\lambda^{-} \\
\lambda^{+} \\
\boldsymbol{s} \\
\boldsymbol{g} \\
\boldsymbol{\gamma}
\end{array}\right) \in \Re^{(2|\mathcal{A}|+3|\mathcal{N}| \times|\mathcal{F}|)} \text { and } \varphi \in \Re^{|\mathcal{F}|} \text {. }
$$


(For notational simplification, we drop the ' in the variable $\varphi$ ). Next, we define the $|\mathcal{A}| \times|\mathcal{A}|$ symmetric positive semidefinite matrix

$$
\Lambda \equiv \Pi^{T} \Xi \Pi
$$

also define two matrices in partitioned form:

$$
\boldsymbol{M}_{\mathrm{oli}} \equiv\left[\begin{array}{cccc}
\boldsymbol{M}_{\lambda} & \boldsymbol{M}_{\lambda s} & \boldsymbol{M}_{\lambda g} & 0 \\
-\left(\boldsymbol{M}_{\lambda s}\right)^{T} & \boldsymbol{M}_{s} & 0 & 0 \\
-\left(\boldsymbol{M}_{\lambda g}\right)^{T} & 0 & 0 & \boldsymbol{I} \\
0 & 0 & -\boldsymbol{I} & 0
\end{array}\right], \quad \boldsymbol{N} \equiv\left[\begin{array}{c}
0 \\
\boldsymbol{J} \\
-\boldsymbol{J} \\
0
\end{array}\right]
$$

where

$$
\begin{aligned}
\boldsymbol{M}_{\lambda} \equiv & {\left[\begin{array}{rr}
\Lambda & -\Lambda \\
-\Lambda & \Lambda
\end{array}\right] \in \Re^{2|\mathcal{A}| \times 2|\mathcal{A}|}, } \\
\boldsymbol{M}_{\lambda s} \equiv & {\left[\begin{array}{rrr}
\Pi^{T} \rho \mathbf{1}_{|\mathcal{N}|}^{T} & \ldots & \Pi^{T} \rho \mathbf{1}_{|\mathcal{N}|}^{T} \\
-\Pi^{T} \rho \mathbf{1}_{|\mathcal{N}|}^{T} & \ldots & -\Pi^{T} \rho \mathbf{1}_{|\mathcal{N}|}^{T}
\end{array}\right] \in \Re^{2|\mathcal{A}| \times(|\mathcal{N}| \times|\mathcal{F}|),} } \\
= & {\left[\begin{array}{r}
\Pi^{T} \rho \\
-\Pi^{T} \rho
\end{array}\right]\left[\begin{array}{lll}
\mathbf{1}_{|\mathcal{N}|}^{T} & \ldots & \mathbf{1}_{|\mathcal{N}|}^{T}
\end{array}\right] } \\
\boldsymbol{M}_{\lambda g} \equiv & {\left[\begin{array}{rrr}
-\Pi^{T} & \ldots & -\Pi^{T} \\
\Pi^{T} & \ldots & \Pi^{T}
\end{array}\right] \in \Re^{2|\mathcal{A}| \times(|\mathcal{N}| \times|\mathcal{F}|)}, } \\
\boldsymbol{M}_{s} \equiv & \omega\left[\begin{array}{rrrr}
\boldsymbol{E} & \boldsymbol{E} & \ldots & \boldsymbol{E} \\
\boldsymbol{E} & \boldsymbol{E} & \ldots & \boldsymbol{E} \\
\vdots & \vdots & \ddots & \vdots \\
\boldsymbol{E} & \boldsymbol{E} & \ldots & \boldsymbol{E}
\end{array}\right] \in \Re^{(|\mathcal{N}| \times|\mathcal{F}|) \times(|\mathcal{N}| \times|\mathcal{F}|)},
\end{aligned}
$$

(with each $\boldsymbol{E}$ being the $|\mathcal{N}| \times|\mathcal{N}|$ matrix of all ones)

$$
\boldsymbol{J} \equiv\left[\begin{array}{cccc}
\mathbf{1}_{|\mathcal{N}|} & 0 & \ldots & 0 \\
0 & \mathbf{1}_{|\mathcal{N}|} & \ldots & 0 \\
\vdots & \vdots & \ddots & \vdots \\
0 & 0 & \ldots & 1_{|\mathcal{N}|}
\end{array}\right] \in \Re^{(|\mathcal{N}| \times|\mathcal{F}|) \times|\mathcal{F}|}
$$


The matrix $\boldsymbol{M}_{\text {oli }}$ is square and of order $(2|\mathcal{A}|+3|\mathcal{N}| \times|\mathcal{F}|)$; the matrix $\boldsymbol{N}$ is rectangular and of order $(2|\mathcal{A}|+3|\mathcal{N}| \times|\mathcal{F}|)$ by $|\mathcal{F}|$. Define a constant vector partitioned in accordance with $M_{\text {oli }}$ :

$$
\boldsymbol{q}_{\mathrm{oli}} \equiv\left(\begin{array}{c}
q^{-} \\
q^{+} \\
-\sigma \mathbf{1}_{|\mathcal{N}| \times|\mathcal{F}|} \\
c \\
\mathrm{CAP}
\end{array}\right) \in \Re^{(2|\mathcal{A}|+3|\mathcal{N}| \times|\mathcal{F}|)}
$$

With the above vectors and matrices, Model I can now be stated as the following mixed NCP:

$$
\begin{aligned}
& 0 \leq \boldsymbol{x} \perp \boldsymbol{q}_{\mathrm{oli}}+\boldsymbol{M}_{\mathrm{oli}} \boldsymbol{x}+\boldsymbol{N} \varphi+\left(\begin{array}{c}
0 \\
0 \\
h_{\mathrm{I}}\left(s, \lambda^{ \pm}\right) \\
0 \\
0
\end{array}\right) \geq 0 \\
& 0=\boldsymbol{N}^{T} \boldsymbol{x} .
\end{aligned}
$$

If not for the nonlinear function $h_{\mathrm{I}}\left(s, \lambda^{ \pm}\right)$, Model I would be a linear complementarity problem (LCP), which is exactly the one treated in [11]. The existence of a solution to (10) relies on bounding the components $h_{\mathrm{I}, f}\left(s, \lambda^{ \pm}\right)$for all $f \in \mathcal{F}$. In turn, this relies on bounding the prices $\pi_{i}$ for $i \in \mathcal{N}$. In Section 6, we show how to obtain the necessary bounds via LCP theory.

\section{Model II}

In this model, each firm takes the arbitrage amounts as input parameters in its profit maximization problem. Specifically, with the price $p_{i}$ given by (7), firm $f$ 's problem is: with $s_{-f i}^{*}\left(=s_{-f i}\right), a_{i}$ and $\pi_{i}$ fixed for 
all $i \in \mathcal{N}$, find $s_{f i}$ and $g_{f i}$ for all $i \in \mathcal{N}$ in order to

$$
\begin{array}{ll}
\text { maximize } & \sum_{i \in \mathcal{N}}\left(\frac{Q_{i}^{0}-s_{f i}-a_{i}+\frac{\alpha_{i}}{\pi_{i}-\alpha_{i}} s_{-f i}^{*}}{\frac{Q_{i}^{0}}{P_{i}^{0}}+\frac{s_{-f i}^{*}}{\pi_{i}-\alpha_{i}}}-w_{i}\right) s_{f i} \\
& -\sum_{i \in \mathcal{N}}\left(c_{f i}-w_{i}\right) g_{f i} \\
\text { subject to } & \sum_{i \in \mathcal{N}}\left(s_{f i}-g_{f i}\right)=0 \\
\text { and } & s_{f i} \geq 0, \quad 0 \leq g_{f i} \leq \operatorname{CAP}_{f i}, \forall(f, i) \in \mathcal{F} \times \mathcal{N} .
\end{array}
$$

Model II is complete with the inclusion of the ISO's problem plus the arbitrage constraint:

$$
\begin{gathered}
\frac{Q_{i}^{0}-s_{f i}-a_{i}+\frac{\alpha_{i}}{\pi_{i}-\alpha_{i}} s_{-f i}^{*}}{\frac{Q_{i}^{0}}{P_{i}^{0}}+\frac{s_{-f i}^{*}}{\pi_{i}-\alpha_{i}}}-w_{i}-p_{H}=0, \quad \forall i \in \mathcal{N}, \\
\sum_{i \in \mathcal{N}} a_{i}=0,
\end{gathered}
$$

the flow balancing equation (5):

$$
y_{i}=\sum_{t \in \mathcal{F}}\left(s_{t i}-g_{t i}\right)+a_{f i}, \quad \forall(f, i) \in \mathcal{F} \times \mathcal{N}
$$

and the price equation

$$
\pi_{i}=w_{i}+p_{f}, \quad \forall i \in \mathcal{N} .
$$

Following a similar derivation as before, we can show that Model II can be formulated as the following NCP:

$$
0 \leq \boldsymbol{x} \perp \boldsymbol{q}_{\mathrm{oli}}+\boldsymbol{M}_{\mathrm{oli}} \boldsymbol{x}+\boldsymbol{N} \varphi+\left(\begin{array}{c}
0 \\
0 \\
h_{\mathrm{II}}\left(s, \lambda^{ \pm}\right) \\
0 \\
0
\end{array}\right) \geq 0
$$


where with $\pi_{i}$ given by $(9), h_{\mathrm{II}}: \Re^{|\mathcal{F}| \times|\mathcal{N}|+2|\mathcal{A}|} \rightarrow \Re^{|\mathcal{F}| \times|\mathcal{N}|}$ is given by

$$
h_{\mathrm{II}, f i}\left(s, \lambda^{ \pm}\right) \equiv \frac{s_{f i}}{\frac{Q_{i}^{0}}{P_{i}^{0}}+\frac{s_{-f i}}{\pi_{i}-\alpha_{i}}}, \quad \forall(f, i) \in \mathcal{F} \times \mathcal{N},
$$

where $\pi_{i}$ is given by (9). The two NCPs (10) and (12) differ in the two closely related nonlinear functions $h_{\mathrm{I}}$ and $h_{\mathrm{II}}$.

\section{Complementarity Theory}

The key to the analysis of Models I and II is the theory of monotone LCPs. This theory in turn yields an existence result of a special variational inequality that is the cornerstone for the existence of solutions to the supply-function based market models. In this section, we present the prerequisite LCP theory and its implications.

We begin by recalling that the LCP range of a matrix $M \in \Re^{n \times n}$, denoted $\mathcal{R}(\boldsymbol{M})$, is the set of all vectors $\boldsymbol{q} \in \Re^{n}$ for which the LCP $(\boldsymbol{q}, \boldsymbol{M})$ has a solution. Our first result pertains to the solutions of an LCP defined by a symmetric positive semidefinite matrix. Although part (a) of this result is known and parts (b) and (c) hold in more general contexts (see [6]) we give a full treatment of the following theorem because it is the basis for the entire subsequent development.

Theorem 1 Let $M \in \Re^{n \times n}$ be a symmetric positive semidefinite matrix.

(a) For every $\boldsymbol{q} \in \mathcal{R}(\boldsymbol{M})$, the solutions of the LCP $(\boldsymbol{q}, \boldsymbol{M})$ are $w$-unique; that is, if $z^{1}$ and $z^{2}$ are any two solutions of the LCP $(\boldsymbol{q}, \boldsymbol{M})$, then $\boldsymbol{M} z^{1}=\boldsymbol{M} z^{2}$. Let $w(\boldsymbol{q})$ denote the common vector $\boldsymbol{q}+\boldsymbol{M} z$ for any solution $z$ of the LCP $(\boldsymbol{q}, \boldsymbol{M})$.

(b) There exists a constant $c>0$ such that

$$
\|w(\boldsymbol{q})\| \leq c\|\boldsymbol{q}\|, \quad \forall \boldsymbol{q} \in \mathcal{R}(\boldsymbol{M})
$$

(c) The function $w: \mathcal{R}(\boldsymbol{M}) \rightarrow \Re^{n}$ is continuous.

Proof. Statement (a) is a well-known result in LCP theory. We next prove (b) by contradiction. Suppose no constant $c$ satisfying (b) exists. There exists a sequence of vectors $\left\{\boldsymbol{q}^{k}\right\} \subset \mathcal{R}(\boldsymbol{M})$ satisfying

$$
\left\|w\left(\boldsymbol{q}^{k}\right)\right\|>k\left\|\boldsymbol{q}^{k}\right\|
$$

for every $k$. We have $w\left(\boldsymbol{q}^{k}\right) \neq 0$ for every $k$ and

$$
\lim _{k \rightarrow \infty} \frac{\boldsymbol{q}^{k}}{\left\|w\left(\boldsymbol{q}^{k}\right)\right\|}=0
$$


Without loss of generality, we may assume that

$$
\lim _{k \rightarrow \infty} \frac{w\left(\boldsymbol{q}^{k}\right)}{\left\|w\left(\boldsymbol{q}^{k}\right)\right\|}=v^{\infty}
$$

for some vector $v^{\infty}$, which must be nonzero. We may further assume that

$$
\operatorname{supp}\left(w\left(\boldsymbol{q}^{k}\right)\right) \equiv\left\{i: w_{i}\left(\boldsymbol{q}^{k}\right)>0\right\}
$$

is the same for all $k$, which we denote $\alpha$. With $\bar{\alpha}$ denoting the complement of $\alpha$ in $\{1, \ldots, n\}$, we have, for every $k$,

$$
\begin{array}{ccc}
0 & = & \boldsymbol{q}_{\bar{\alpha}}^{k}+\boldsymbol{M}_{\bar{\alpha} \bar{\alpha}} z_{\bar{\alpha}}^{k} \\
w_{\alpha}\left(\boldsymbol{q}^{k}\right) & =\boldsymbol{q}_{\alpha}^{k}+\boldsymbol{M}_{\alpha \bar{\alpha}} z_{\bar{\alpha}}^{k}>0
\end{array}
$$

for some vector $z_{\bar{\alpha}}^{k} \geq 0$. Dividing by $\left\|w\left(\boldsymbol{q}^{k}\right)\right\|$, we deduce the existence of a nonnegative vector $z_{\bar{\alpha}}^{\infty}$, which is not necessarily related to the sequence $\left\{z_{\bar{\alpha}}^{k}\right\}$, such that

$$
\begin{gathered}
0=\boldsymbol{M}_{\bar{\alpha} \bar{\alpha}} z_{\bar{\alpha}}^{\infty} \\
v_{\alpha}^{\infty}=M_{\alpha \bar{\alpha}} z_{\bar{\alpha}}^{\infty} .
\end{gathered}
$$

Since $M$ is symmetric positive semidefinite, the above implies $v_{\bar{\alpha}}^{\infty}$, thus $v^{\infty}$, is equal to zero. This contradiction establishes part (b).

To prove part (c), let $\left\{q^{k}\right\} \subset \mathcal{R}(\boldsymbol{M})$ converge to a limit vector $q^{\infty}$ which must necessarily belong to $\mathcal{R}(\boldsymbol{M})$ because the LCP range is a closed cone. For each $k$, let $z^{k} \in \operatorname{SOL}\left(q^{k}, M\right)$ be such that $w\left(q^{k}\right)=$ $q^{k}+M z^{k}$. The sequence $\left\{w\left(q^{k}\right)\right\}$ is bounded; moreover, if $w^{\infty}$ is any accumulation point of this $w$-sequence, then using the complementary cone argument, as done in the proof of part (b), we deduce the existence of a solution $z^{\infty} \in \operatorname{SOL}\left(q^{\infty}, \boldsymbol{M}\right)$ such that $w^{\infty}=q^{\infty}+\boldsymbol{M} z^{\infty}$. This is enough to show by part (a) that the sequence $\left\{w\left(q^{k}\right)\right\}$ has a unique accumulation point which is equal to $w\left(q^{\infty}\right)$. Therefore the continuity of the map $w(q)$ at every vector $q \in \mathcal{R}(\boldsymbol{M})$ follows.

Q.E.D.

Our goal is to apply the above theorem to the matrix

$$
\boldsymbol{M}_{\lambda}=\left[\begin{array}{rr}
\Lambda & -\Lambda \\
-\Lambda & \Lambda
\end{array}\right]=\left[\begin{array}{c}
\Pi^{T} \\
-\Pi^{T}
\end{array}\right] \Xi\left[\begin{array}{ll}
\Pi & -\Pi] .
\end{array}\right.
$$

For this purpose, we derive a corollary of Theorem 1 pertaining to a symmetric positive definite matrix of the above form.

Corollary 1 Let $M \equiv A^{T} E A$, where $E$ is a symmetric positive semidefinite $m \times m$ matrix and $A$ is an arbitrary $m \times n$ matrix. 
(a) For every $\boldsymbol{q} \in \mathcal{R}(\boldsymbol{M})$, if $z^{1}$ and $z^{2}$ are any two solutions of the LCP $(\boldsymbol{q}, \boldsymbol{M})$, then $E A z^{1}=E A z^{2}$. Let $\tilde{w}(\boldsymbol{q})$ denote the common vector $E A z$ for any solution $z$ of the LCP $(\boldsymbol{q}, \boldsymbol{M})$.

(b) There exists a constant $c^{\prime}>0$ such that

$$
\|\tilde{w}(\boldsymbol{q})\| \leq c^{\prime}\|\boldsymbol{q}\|, \quad \forall \boldsymbol{q} \in \mathcal{R}(\boldsymbol{M}) .
$$

(c) The function $\tilde{w}: \mathcal{R}(\boldsymbol{M}) \rightarrow \Re^{n}$ is continuous.

Proof. We note that for any nonzero symmetric positive semidefinite $\boldsymbol{M}$, we have

$$
\frac{1}{\lambda_{\min }^{+}(\boldsymbol{M})}\|\boldsymbol{M} z\|^{2} \geq z^{T} \boldsymbol{M} z \geq \frac{1}{\lambda_{\max }(\boldsymbol{M})}\|\boldsymbol{M} z\|^{2}, \quad \forall z \in \Re^{n},
$$

where $\lambda_{\min }^{+}(\boldsymbol{M})$ is the smallest positive eigenvalue of $\boldsymbol{M}$ and $\lambda_{\max }(\boldsymbol{M})$ is the largest eigenvalue of $\boldsymbol{M}$. With $\boldsymbol{M}=A^{T} E A$, it follows that

$$
M z=0 \Leftrightarrow E A z=0 .
$$

Hence, for every $\boldsymbol{q} \in \mathcal{R}(\boldsymbol{M}), E A z$ is a constant for all solutions of the LCP $(\boldsymbol{q}, \boldsymbol{M})$. Moreover, there exists a scalar $c>0$ such that for every $\boldsymbol{q} \in \mathcal{R}(\boldsymbol{M})$,

$$
\|\boldsymbol{M} z\| \leq(1+c)\|\boldsymbol{q}\|,
$$

for every solution $z$ of the LCP $(\boldsymbol{q}, \boldsymbol{M})$. Since

$$
\frac{1}{\lambda_{\min }^{+}(\boldsymbol{M})}\|\boldsymbol{M} z\|^{2} \geq z^{T} \boldsymbol{M} z=(A z)^{T} E A z \geq \frac{1}{\lambda_{\max }(E)}\|E A z\|^{2}
$$

for all $z \in \Re^{n}$, part (b) of the corollary follows readily. The proof of part (c) is very similar to that of the same part in Theorem 1. Q.E.D.

It can be shown, using the theory of piecewise affine functions, that both functions $w(q)$ and $\tilde{w}(q)$ are Lipschitz continuous on $\mathcal{R}(M)$. Since this Lipschitz continuity property is not needed in the subsequent analysis, we omit the details.

\subsection{An existence result for a special VI}

In what follows, we establish an existence result for a linearly constrained variational inequality (VI) of a special kind. This result will subsequently be applied to Models I and II of power market equilibria. The setup of the result is a VI $(K, F)$, where $K$ is the Cartesian product 
of two polyhedra $K_{1} \subseteq \Re^{n_{1}}$ and $K_{2} \subset \Re^{n_{2}}$ with $K_{2}$ being compact. The mapping $F$ is of the form: for $(x, y) \in \Re^{n_{1}+n_{2}}$,

$$
F(x, y) \equiv\left(\begin{array}{c}
q \\
r
\end{array}\right)+\left[\begin{array}{ll}
M_{11} & M_{12} \\
M_{21} & M_{22}
\end{array}\right]\left(\begin{array}{l}
x \\
y
\end{array}\right)+\left(\begin{array}{c}
0 \\
h(y)
\end{array}\right),
$$

where $h: \Re^{n_{2}} \rightarrow \Re^{n_{2}}$ is a continuous function and the matrix

$$
M \equiv\left[\begin{array}{ll}
M_{11} & M_{12} \\
M_{21} & M_{22}
\end{array}\right]
$$

is positive semidefinite (not necessarily symmetric). In the following result, an AVI is a VI defined by an affine pair $(\hat{K}, \hat{F})$, i.e., $\hat{K}$ is a polyhedron and $\hat{F}$ is an affine map. (We refer the reader to the monograph [6] for a comprehensive treatment of the finite-dimensional variational inequalities and complementarity problems.)

Proposition 1 In addition to the above setting, assume that for all $\hat{y} \in K_{2}$, the AVI $\left(K, F^{\hat{y}}\right)$ has a solution, where

$$
F^{\hat{y}}(x, y) \equiv\left(\begin{array}{c}
q \\
r+h(\hat{y})
\end{array}\right)+\left[\begin{array}{ll}
M_{11} & M_{12} \\
M_{21} & M_{22}
\end{array}\right]\left(\begin{array}{l}
x \\
y
\end{array}\right), \quad(x, y) \in \Re^{n_{1}+n_{2}} .
$$

The VI $(K, F)$ has a solution.

Proof. We apply Kakutani's fixed-point theorem to the set-valued mapping $\Gamma: K_{2} \rightarrow K_{2}$ defined as follows. For each $\hat{y} \in K_{2}, \Gamma(\hat{y})$ consists of all vectors $y \in K_{2}$ for which there exists a vector $x \in K_{1}$ such that the pair $(x, y)$ solves the VI $\left(K, F^{\hat{y}}\right)$. Clearly, $\Gamma(\hat{y})$ is a nonempty subset of $K_{2} ; \Gamma(\hat{y})$ is convex because if $y^{1}$ and $y^{2}$ are any two elements in $\Gamma(\hat{y})$ and $x^{1}$ and $x^{2}$ are such that $\left(x^{i}, y^{i}\right) \in \operatorname{SOL}\left(K, F^{\hat{y}}\right)$ for $i=1,2$, then $\tau\left(x^{1}, y^{1}\right)+(1-\tau)\left(x^{2}, y^{2}\right)$ remains a solution of the VI $\left(K, F^{\hat{y}}\right)$ for all scalars $\tau \in(0,1)$, by the positive semidefiniteness of the matrix $\boldsymbol{M}$. We next verify that $\Gamma$ is a closed map. For this purpose, let $\left\{\hat{y}^{k}\right\}$ be a sequence of vectors in $K_{2}$ converging to a vector $\hat{y}^{\infty}$ in $K_{2}$ and for each $k$ let $\left(x^{k}, y^{k}\right)$ be a solution of the VI $\left(K, F^{\hat{y}^{k}}\right)$ such that the sequence $\left\{y^{k}\right\}$ converges to a vector $y^{\infty}$. We need to show the existence of a vector $x^{\infty}$ such that the pair $\left(x^{\infty}, y^{\infty}\right)$ solves the VI $\left(K, F^{\hat{y}^{\infty}}\right)$. Write

$$
K_{1} \equiv\left\{x \in \Re^{n_{1}}: A x \leq b\right\}
$$

and

$$
K_{2} \equiv\left\{y \in \Re^{n_{2}}: C y \leq d\right\}
$$


For each $k$, there exist multipliers $\mu^{k}$ and $\eta^{k}$ such that

$$
\begin{aligned}
&\left(\begin{array}{c}
q \\
r+h\left(\hat{y}^{k}\right)
\end{array}\right)+ {\left[\begin{array}{ll}
M_{11} & M_{12} \\
M_{21} & M_{22}
\end{array}\right]\left(\begin{array}{c}
x^{k} \\
y^{k}
\end{array}\right)+\left(\begin{array}{c}
A^{T} \mu^{k} \\
C^{T} \eta^{k}
\end{array}\right)=0 } \\
& 0 \leq \mu^{k} \quad \perp A x^{k}-b \leq 0 \\
& 0 \leq \eta^{k} \perp C y^{k}-d \leq 0 .
\end{aligned}
$$

Again by a standard complementary cone argument, we can deduce the existence of $\mu^{\infty}, \eta^{\infty}$, and $x^{\infty}$, which are not necessarily the limits of the sequences $\left\{\mu^{k}\right\},\left\{\eta^{k}\right\}$ and $\left\{x^{k}\right\}$, respectively, such that

$$
\begin{aligned}
\left(\begin{array}{c}
q \\
r+h\left(\hat{y}^{\infty}\right)
\end{array}\right)+ & {\left[\begin{array}{ll}
M_{11} & M_{12} \\
M_{21} & M_{22}
\end{array}\right]\left(\begin{array}{c}
x^{\infty} \\
y^{\infty}
\end{array}\right)+\left(\begin{array}{c}
A^{T} \mu^{\infty} \\
C^{T} \eta^{\infty}
\end{array}\right)=0 } \\
0 & \leq \mu^{\infty} \perp A x^{\infty}-b \leq 0 \\
0 & \leq \eta^{\infty} \perp C y^{\infty}-d \leq 0 .
\end{aligned}
$$

This establishes that $\Gamma$ is a closed map. In particular, it follows that $\Gamma(\hat{y})$ is a closed subset of $K_{2}$ for all $\hat{y}$ in $K_{2}$. Thus $\Gamma$ satisfies all the assumptions required for the applicability of Kakutani's fixed-point theorem. By this theorem, $\Gamma$ has a fixed point, which can easily be seen to be a solution of the VI $(K, F)$.

Q.E.D.

\section{Properties of Models I and II}

Returning to the mixed NCPs (10) and (12), we consider the following LCP in the variables $\lambda^{\mp}$, parameterized by $S$ and $\boldsymbol{g}$ :

$$
\begin{aligned}
& 0 \leq \lambda^{-} \perp q^{-}+\Lambda\left(\lambda^{-}-\lambda^{+}\right)+\Pi^{T}\left(\rho S-\sum_{f \in \mathcal{F}} g_{f}\right) \geq 0 \\
& 0 \leq \lambda^{+} \perp q^{+}+\Lambda\left(\lambda^{+}-\lambda^{-}\right)-\Pi^{T}\left(\rho S-\sum_{f \in \mathcal{F}} g_{f}\right) \geq 0 .
\end{aligned}
$$

We want to derive a sufficient condition under which the above LCP will have a solution for all "feasible" sales and generations. Specifically, let

$$
\begin{aligned}
Y \equiv \prod_{f \in \mathcal{F}}\left\{\quad\left(s_{f}, g_{f}\right) \in \Re_{+}^{2|\mathcal{N}|}:\right. \\
\left.\quad \sum_{i \in \mathcal{N}}\left(s_{f i}-g_{f i}\right)=0, g_{f i} \leq \mathrm{CAP}_{f i}, \forall(f, i) \in \mathcal{F} \times \mathcal{N}\right\},
\end{aligned}
$$


be the set of such sales and generations. The set $Y$ is a compact polyhedron in $\Re^{2|\mathcal{F}| \times|\mathcal{N}|}$. We have for all pairs $(\boldsymbol{s}, \boldsymbol{g}) \in Y$ and every $j \in \mathcal{N}$,

$$
\rho_{j} S-\sum_{f \in \mathcal{F}} g_{f j}=-\sum_{f \in \mathcal{N}} \sum_{i \in \mathcal{N}}\left(\delta_{i j}-\rho_{j}\right) g_{f i} .
$$

Thus

$$
\begin{aligned}
& \sum_{j \in \mathcal{N}} \rho_{j} \operatorname{PDF}_{j k} S-\sum_{j \in \mathcal{N}} \sum_{f \in \mathcal{F}} \operatorname{PDF}_{j k} g_{f j} \\
& =-\sum_{f \in \mathcal{F}} \sum_{i \in \mathcal{N}} \sum_{j \in \mathcal{N}} \operatorname{PDF}_{j k}\left(\delta_{i j}-\rho_{j}\right) g_{f i} \\
& =-\sum_{f \in \mathcal{F}} \sum_{i \in \mathcal{N}}\left(\sum_{j \in \mathcal{N}} \operatorname{PDF}_{j k} \zeta_{i j}\right) \frac{P_{i}^{0}}{Q_{i}^{0}} g_{f i} .
\end{aligned}
$$

Therefore, the LCP (14) can be written as:

$$
\begin{aligned}
& 0 \leq\left(\begin{array}{c}
\lambda^{-} \\
\lambda^{+}
\end{array}\right) \perp\left(\begin{array}{c}
q^{-} \\
q^{+}
\end{array}\right)-\left[\begin{array}{c}
\Pi^{T} \\
-\Pi^{T}
\end{array}\right] \Xi D \sum_{f \in \mathcal{F}} g_{f} \\
& +\left[\begin{array}{c}
\Pi^{T} \\
-\Pi^{T}
\end{array}\right] \Xi\left[\begin{array}{ll}
\Pi & -\Pi
\end{array}\right]\left(\begin{array}{l}
\lambda^{-} \\
\lambda^{+}
\end{array}\right) \geq 0,
\end{aligned}
$$

where $D$ is the $|\mathcal{N}| \times|\mathcal{N}|$ diagonal matrix with diagonal entries $P_{i}^{0} / Q_{i}^{0}$.

Proposition 2 If there exists a vector $\lambda \in \Re^{|\mathcal{A}|}$ satisfying

$$
-T^{-} \leq \Pi^{T} \Xi P^{0}+\Pi^{T} \Xi \Pi \lambda \leq T^{+},
$$

then the LCP (14) has a solution $\lambda^{\mp}$ for every pair $(\boldsymbol{s}, \boldsymbol{g}) \in Y$.

Proof. The LCP (15) is of the form:

$$
0 \leq \boldsymbol{z} \perp \boldsymbol{q}+A^{T} E \boldsymbol{r}+A^{T} E A \boldsymbol{z} \geq 0,
$$

where $E$ is a symmetric positive semidefinite matrix. It follows from LCP theory that if there exists a vector $\hat{\boldsymbol{z}}$ satisfying $\boldsymbol{q}+A^{T} E \hat{\boldsymbol{z}} \geq 0$, then the LCP $\left(\boldsymbol{q}+A^{T} E \boldsymbol{r}, \boldsymbol{M}\right)$, where $\boldsymbol{M} \equiv A^{T} E A$, has a solution for all vectors $r$.

Q.E.D.

Throughout the following discussion, we assume that condition (16) holds. Thus the LCP (15) has a solution for all vectors $\boldsymbol{g}$. Moreover, 
specializing Corollary 1 to the matrix $\boldsymbol{M}_{\lambda}$, we deduce that for any vector $\boldsymbol{g}$, if $\left(\lambda^{-, i}, \lambda^{+, i}\right)$ for $i=1,2$ are any two solutions of (15), we have

$$
\Xi \Pi\left(\lambda^{+, 1}-\lambda^{-, 1}\right)=\Xi \Pi\left(\lambda^{+, 2}-\lambda^{-, 2}\right) .
$$

Furthermore, if $\Phi(\boldsymbol{g})$ denotes this common vector, then $\Phi$ is a Lipschitz continuous function from $\Re^{|\mathcal{F}| \times|\mathcal{N}|}$ into $\Re^{|\mathcal{N}|}$. In terms of this function, we have

$$
\pi_{i}=\sigma-\omega S+\Phi_{i}(\boldsymbol{g}), \quad \forall i \in \mathcal{N},
$$

which shows that $\pi_{i}$ is a function of the total sales $S$ and the generations $\boldsymbol{g}$. Since each $\Phi$ is continuous and $Y$ is compact, it follows that for each $i \in \mathcal{N}$, the scalar

$$
\varsigma_{i} \equiv \min \left\{\sigma-\omega S+\Phi_{i}(\boldsymbol{g}):(\boldsymbol{s}, \boldsymbol{g}) \in Y\right\}
$$

is finite. Therefore, if the intercepts $\alpha_{i}$ satisfy

$$
\alpha_{i}<\varsigma_{i}, \quad \forall i \in \mathcal{N},
$$

then the denominators in

$$
h_{\mathrm{I}, f i}(\boldsymbol{s}, \boldsymbol{g}) \equiv\left(\sum_{j \in \mathcal{N}} s_{f j}\right) /\left(\sum_{j \in \mathcal{N}}\left(\frac{Q_{j}^{0}}{P_{j}^{0}}+\frac{s_{-f j}}{\pi_{j}-\alpha_{j}}\right)\right)
$$

and

$$
h_{\mathrm{II}, f i}(\boldsymbol{s}, \boldsymbol{g}) \equiv s_{f i} /\left(\frac{Q_{i}^{0}}{P_{i}^{0}}+\frac{s_{-f i}}{\pi_{i}-\alpha_{i}}\right)
$$

are positive for all $(\boldsymbol{s}, \boldsymbol{g}) \in Y$ and all $f \in \mathcal{F}$. Notice that as a result of (17), we can replace the dependence on $\lambda^{ \pm}$in the two functions $h_{\mathrm{I}}$ and $h_{\mathrm{II}}$ by the dependence on $\boldsymbol{g}$ instead. The computation of each scalar $\varsigma_{i}$ requires the solution of an mathematical program with equilibrium constraints [10] that has a linear objective function and a parametric, monotone LCP constraint.

\subsection{Existence of solutions}

Both NCPs (10) and (12) are equivalent to a VI of the type considered in Proposition 1. More specifically, define the following principal submatrix of $M_{\text {oli }}$ by removing the last row and column:

$$
\tilde{\boldsymbol{M}}_{\mathrm{oli}} \equiv\left[\begin{array}{ccc}
\boldsymbol{M}_{\lambda} & \boldsymbol{M}_{\lambda s} & \boldsymbol{M}_{\lambda g} \\
-\left(\boldsymbol{M}_{\lambda s}\right)^{T} & \boldsymbol{M}_{s} & 0 \\
-\left(\boldsymbol{M}_{\lambda g}\right)^{T} & 0 & 0
\end{array}\right]
$$


the matrix $\tilde{M}_{\text {oli }}$ is of order $(2|\mathcal{A}|+2|\mathcal{N}| \times|\mathcal{F}|)$. Define a reduced vector $\tilde{\boldsymbol{q}}_{\text {oli }}$ accordingly:

$$
\tilde{\boldsymbol{q}}_{\text {oli }} \equiv\left(q^{-}, q^{+}, \quad-\sigma \mathbf{1}_{|\mathcal{N}| \times|\mathcal{F}|}, c\right)^{T} \in \Re^{(2|\mathcal{A}|+2|\mathcal{N}| \times|\mathcal{F}|)} .
$$

Let $n_{1} \equiv 2|\mathcal{A}|$ and $n_{2} \equiv 2|\mathcal{N}| \times|\mathcal{F}|$. Identify the vectors $x$ and $y$ with $\lambda^{\mp}$ and $(\boldsymbol{s}, \boldsymbol{g})$, respectively, the constant vector $(q, r)$ with $\tilde{\boldsymbol{q}}_{\text {oli }}$, the matrix $\boldsymbol{M}$ with $\tilde{M}_{\text {oli }}$ and the function $h(y)$ with either

$$
\left(\begin{array}{c}
h_{\mathrm{I}}(\boldsymbol{s}, \boldsymbol{g}) \\
0
\end{array}\right) \quad \text { or } \quad\left(\begin{array}{c}
h_{\mathrm{II}}(\boldsymbol{s}, \boldsymbol{g}) \\
0
\end{array}\right) \text {. }
$$

Furthermore, let $K_{1}$ be the nonnegative orthant of $\Re^{2|\mathcal{A}|}$ and $K_{2}$ be the set $Y$ :

$$
\begin{aligned}
K_{2} \equiv \prod_{f \in \mathcal{F}}\left\{\quad\left(s_{f}, g_{f}\right) \in \Re_{+}^{2|\mathcal{N}|}:\right. \\
\\
\left.\sum_{i \in \mathcal{N}}\left(s_{f i}-g_{f i}\right)=0, g_{f i} \leq \operatorname{CAP}_{f i}, \forall(f, i) \in \mathcal{F} \times \mathcal{N}\right\} .
\end{aligned}
$$

Under the above identifications, models I and II can therefore be formulated as the VI $\left(K_{1} \times K_{2}, F\right)$, where $F$ is given by (13). We can readily apply Proposition 1 to establish the following existence result for the two models.

Theorem 2 Suppose that there exists a vector $\lambda \in \Re^{|\mathcal{A}|}$ satisfying (16). If

$$
\alpha_{i}<\min \left\{\sigma-\omega S+\Phi_{i}(\boldsymbol{g}):(\boldsymbol{s}, \boldsymbol{g}) \in Y\right\}, \quad \forall i \in \mathcal{N},
$$

then solutions exist to Models I and II.

Proof. Under the assumption on the intercepts $\alpha_{i}$, the function $h(\boldsymbol{s}, \boldsymbol{g})$ is well defined on the set $K_{2}$. For every $\hat{y} \equiv(\hat{\boldsymbol{s}}, \hat{\boldsymbol{g}}) \in K_{2}$, the VI $\left(K, F^{\hat{y}}\right)$ is equivalent to the mixed LCP in the variable $(\boldsymbol{x}, \varphi)$ :

$$
\begin{aligned}
& 0 \leq \boldsymbol{x} \perp \boldsymbol{q}_{\mathrm{oli}}+\boldsymbol{M}_{\mathrm{oli}} \boldsymbol{x}+\boldsymbol{N} \varphi+(0,0, h(\hat{\boldsymbol{s}}, \hat{\boldsymbol{g}}))^{T} \geq 0 \\
& 0=\boldsymbol{N}^{T} \boldsymbol{x} .
\end{aligned}
$$

This mixed LCP is clearly feasible and monotone. It therefore has a solution. The existence of solutions to Models I and II follows readily from Proposition 1.

Q.E.D.

The next result identifies two important properties of the solutions to Model II. It shows in particular that Model I can be solved by solving Model II. 
Theorem 3 Under the assumptions of Theorem 2 , if $\left(\lambda^{\mp}, \boldsymbol{s}, \boldsymbol{g}, \varphi\right)$ is a solution to Model II, then $\forall i \neq j$ and $\forall f \in \mathcal{F}$

$$
s_{f i} /\left(\frac{Q_{i}^{0}}{P_{i}^{0}}+\frac{s_{-f i}}{\pi_{i}-\alpha_{i}}\right)=s_{f j} /\left(\frac{Q_{j}^{0}}{P_{j}^{0}}+\frac{s_{-f j}}{\pi_{j}-\alpha_{j}}\right) .
$$

Therefore every solution to Model II is a solution to Model I.

Proof. It suffices to show that for all $i \neq j$ and all $f \in \mathcal{F}$,

$$
s_{f i} /\left(\frac{Q_{i}^{0}}{P_{i}^{0}}+\frac{s_{-f i}}{\pi_{i}-\alpha_{i}}\right) \geq s_{f j} /\left(\frac{Q_{j}^{0}}{P_{j}^{0}}+\frac{s_{-f j}}{\pi_{j}-\alpha_{j}}\right) .
$$

This is because by reversing the role of $i$ and $j$, we obtain the reverse inequality and equality therefore must hold. The above inequality is clearly valid if $s_{f j}=0$. So we may assume that $s_{f j}>0$. By complementarity, we have

$$
\begin{aligned}
\frac{s_{f j}}{\frac{Q_{j}^{0}}{P_{j}^{0}}+\frac{s_{-f j}}{\pi_{j}-\alpha_{j}}} & =\sigma-\omega S-\varphi_{f}-\sum_{k \in \mathcal{A}}\left[\sum_{j^{\prime} \in \mathcal{N}} \rho_{j^{\prime}} \mathrm{PDF}_{j^{\prime} k}\right]\left(\lambda_{k}^{+}-\lambda_{k}^{-}\right) \\
& \leq \frac{s_{f i}}{\frac{Q_{i}^{0}}{P_{i}^{0}}+\frac{s_{-f i}}{\pi_{i}-\alpha_{i}}}
\end{aligned}
$$

This establishes the first assertion of the theorem. To prove the second assertion, we note that by what has just been proved, it follows that if $\left(\lambda^{\mp}, \boldsymbol{s}, \boldsymbol{g}, \varphi\right)$ is a solution to Model II, then we must have

$$
s_{f i} /\left(\frac{Q_{i}^{0}}{P_{i}^{0}}+\frac{s_{-f i}}{\pi_{i}-\alpha_{i}}\right)=\sum_{j \in \mathcal{N}} s_{f j} / \sum_{j \in \mathcal{N}}\left[\frac{Q_{j}^{0}}{P_{j}^{0}}+\frac{s_{-f j}}{\pi_{j}-\alpha_{j}}\right],
$$

This shows that $\left(\lambda^{\mp}, \boldsymbol{s}, \boldsymbol{g}\right)$ is also a solution to Model I.

Q.E.D.

\subsection{Uniqueness in Model II}

In this subsection, we show that if each price intercept $\alpha_{i}$ is suitably restricted, then the firms' sales in the market model II are unique. The cornerstone to this uniqueness property of the model solutions is the 
expression (9). Based on this expression, we show that the mapping

$$
\boldsymbol{F}_{\mathrm{II}}(\boldsymbol{x}, \varphi) \equiv\left(\begin{array}{c}
\boldsymbol{q}_{\mathrm{oli}} \\
0
\end{array}\right)+\left[\begin{array}{cc}
\boldsymbol{M}_{\mathrm{oli}} & \boldsymbol{N} \\
-\boldsymbol{N} & 0
\end{array}\right]\left(\begin{array}{c}
\boldsymbol{x} \\
\varphi
\end{array}\right)+\left(\begin{array}{c}
0 \\
0 \\
h_{\mathrm{II}}\left(s, \lambda^{ \pm}\right) \\
0 \\
0
\end{array}\right)
$$

is monotone. Throughout the following analysis, we restrict the pair $\left(S, \lambda^{ \pm}\right)$so that

$$
\sigma-\omega S+\sum_{j \in \mathcal{N}} \sum_{k \in \mathcal{A}}\left(\delta_{i j}-\rho_{j}\right) \operatorname{PDF}_{j k}\left(\lambda_{k}^{+}-\lambda_{k}^{-}\right)>\alpha_{i}, \quad \forall i \in \mathcal{N} .
$$

To establish the desired monotonicity of $\boldsymbol{F}_{\mathrm{II}}$, we first compute the Jacobian matrix of the function $h_{\mathrm{II}}\left(s, \lambda^{ \pm}\right)$.

We begin by noting the following partial derivatives:

$$
\frac{\partial \pi_{i}}{\partial s_{f i^{\prime}}}=-\omega, \quad \forall f \in \mathcal{F}, i, i^{\prime} \in \mathcal{N}
$$

and

$$
\frac{\partial \pi_{i}}{\partial \lambda_{k}^{ \pm}}= \pm \frac{P_{i}^{0}}{Q_{i}^{0}} \sum_{j \in \mathcal{N}} \zeta_{i j} \mathrm{PDF}_{j k} \quad \forall i \in \mathcal{N}, k \in \mathcal{A} .
$$

Next, recalling that

$$
h_{\mathrm{II}, f i}\left(s, \lambda^{ \pm}\right)=\frac{s_{f i}}{\frac{Q_{i}^{0}}{P_{i}^{0}}+\frac{s_{-f i}}{\pi_{i}-\alpha_{i}}}, \quad \forall f \in \mathcal{F}, i \in \mathcal{N},
$$

we have for all $f \in \mathcal{F}$,

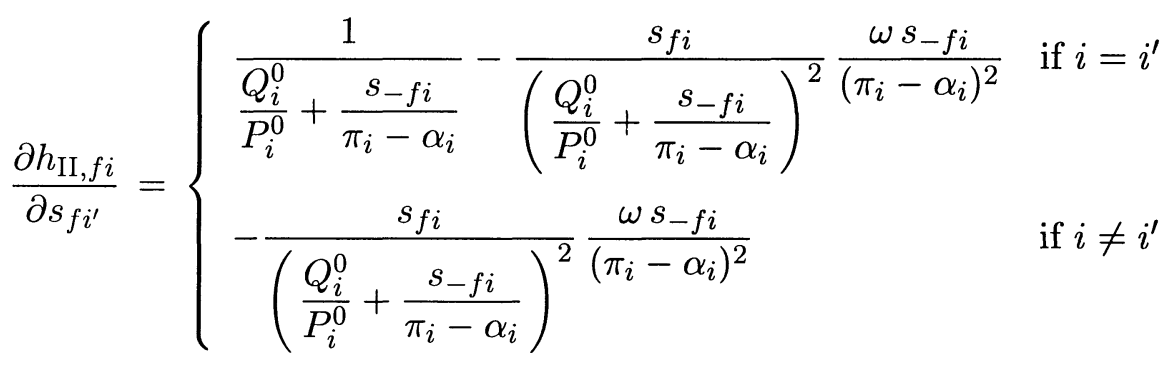


and for all $f \neq f^{\prime}$,

$$
\frac{\partial h_{\mathrm{II}, f i}}{\partial s_{f^{\prime} i^{\prime}}}= \begin{cases}-\frac{s_{f i}}{\left(\frac{Q_{i}^{0}}{P_{i}^{0}}+\frac{s_{-f i}}{\pi_{i}-\alpha_{i}}\right)^{2}}\left(\frac{1}{\pi_{i}-\alpha_{i}}+\frac{\omega s_{-f i}}{\left(\pi_{i}-\alpha_{i}\right)^{2}}\right) & \text { if } i=i^{\prime} \\ -\frac{s_{f i}}{\left(\frac{Q_{i}^{0}}{P_{i}^{0}}+\frac{s_{-f i}}{\pi_{i}-\alpha_{i}}\right)^{2}} \frac{\omega s_{-f i}}{\left(\pi_{i}-\alpha_{i}\right)^{2}} & \text { if } i \neq i^{\prime} .\end{cases}
$$

Moreover, for all $f \in \mathcal{F}, i \in \mathcal{N}$ and $k \in \mathcal{A}$,

$$
\frac{\partial h_{\mathrm{II}, f i}}{\partial \lambda_{k}^{ \pm}}=\mp \frac{s_{f i}}{\left(\frac{Q_{i}^{0}}{P_{i}^{0}}+\frac{s_{-f i}}{\pi_{i}-\alpha_{i}}\right)^{2}} \frac{s_{-f i}}{\left(\pi_{i}-\alpha_{i}\right)^{2}} \frac{P_{i}^{0}}{Q_{i}^{0}} \sum_{j \in \mathcal{N}} \zeta_{i j} \mathrm{PDF}_{j k}
$$

Therefore, the Jacobian matrix of $h_{\mathrm{II}}\left(s, \lambda^{ \pm}\right)$has the following partitioned form:

$$
\left[\begin{array}{ccccc}
B_{1}^{\lambda^{-}} & B_{1}^{\lambda^{+}} & A_{11}^{s} & \cdots & A_{1|\mathcal{F}|}^{s} \\
\vdots & \vdots & \vdots & \ddots & \vdots \\
B_{|\mathcal{F}|}^{\lambda^{-}} & B_{|\mathcal{F}|}^{\lambda^{+}} & A_{|\mathcal{F}| 1}^{s} & \cdots & A_{|\mathcal{F}||\mathcal{F}|}^{s}
\end{array}\right]
$$

where each $A_{f f^{\prime}}^{s}$ is an $|\mathcal{N}| \times|\mathcal{N}|$ matrix with entries

$$
\left(A_{f f^{\prime}}^{s}\right)_{i i^{\prime}}=\frac{\partial h_{\mathrm{II} f i}}{\partial s_{f^{\prime} i^{\prime}}}, \quad \forall i, i^{\prime} \in \mathcal{N}
$$

and each $B_{f}^{\lambda^{ \pm}}$is an $|\mathcal{N}| \times|\mathcal{A}|$ matrix with entries

$$
\left(B_{f}^{\lambda^{ \pm}}\right)_{i k}=\frac{\partial h_{\mathrm{II} f i}}{\partial \lambda_{k}^{ \pm}}, \quad \forall i \in \mathcal{N}, k \in \mathcal{A} .
$$

Consequently, the Jacobian matrix of $\boldsymbol{F}_{\mathrm{II}}(\boldsymbol{x}, \varphi)$ can be written as the sum of two matrices $L_{1}$ and $L_{2}$, where

$$
L_{1} \equiv\left[\begin{array}{ccccccc}
\Lambda & -\Lambda & 0 & \cdots & 0 & 0 & 0 \\
-\Lambda & \Lambda & 0 & \cdots & 0 & 0 & 0 \\
B_{1}^{\lambda^{-}} & B_{1}^{\lambda^{+}} & A_{11}^{s} & \cdots & A_{1|\mathcal{F}|}^{s} & 0 & 0 \\
\vdots & \vdots & \vdots & \ddots & \vdots & & \\
B_{|\mathcal{F}|}^{\lambda^{-}} & B_{|\mathcal{F}|}^{\lambda^{+}} & A_{|\mathcal{F}| 1}^{s} & \cdots & A_{|\mathcal{F}| \mathcal{F} \mid}^{s} & 0 & 0 \\
0 & 0 & 0 & 0 & 0 & 0 & 0 \\
0 & 0 & 0 & 0 & 0 & 0 & 0
\end{array}\right]
$$


and

$$
L_{2} \equiv\left[\begin{array}{ccccc}
0 & \boldsymbol{M}_{\lambda s} & \boldsymbol{M}_{\lambda g} & 0 & 0 \\
-\left(\boldsymbol{M}_{\lambda s}\right)^{T} & \boldsymbol{M}_{s} & 0 & 0 & \boldsymbol{J} \\
-\left(\boldsymbol{M}_{\lambda g}\right)^{T} & 0 & 0 & \boldsymbol{I} & -\boldsymbol{J} \\
0 & 0 & -\boldsymbol{I} & 0 & 0 \\
0 & -\boldsymbol{J} & \boldsymbol{J} & 0 & 0
\end{array}\right]
$$

The matrix $L_{2}$ is skew-symmetric, thus positive semidefinite. To show that $L_{1}$ is also positive semidefinite, recall that $\Lambda=\Pi^{T} \Xi \Pi$. Furthermore, we have

$$
B_{f}^{\lambda^{ \pm}}= \pm D_{f} \Xi \Pi, \quad \forall f \in \mathcal{F},
$$

for some $|\mathcal{N}| \times|\mathcal{N}|$ diagonal matrix $D_{f}$ with

$$
\left(D_{f}\right)_{i i} \equiv \frac{s_{f i}}{\left(\frac{Q_{i}^{0}}{P_{i}^{0}}+\frac{s_{-f i}}{\pi_{i}-\alpha_{i}}\right)^{2}} \frac{s_{-f i}}{\left(\pi_{i}-\alpha_{i}\right)^{2}} \frac{P_{i}^{0}}{Q_{i}^{0}}, \quad \forall i \in \mathcal{N} .
$$

Let

$$
\boldsymbol{A} \equiv\left[\begin{array}{ccc}
A_{11}^{s} & \cdots & A_{1|\mathcal{F}|}^{s} \\
\vdots & \ddots & \vdots \\
A_{|\mathcal{F}| 1}^{s} & \cdots & A_{|\mathcal{F}| \mathcal{F} \mid}^{s}
\end{array}\right] \quad \text { and } \quad \boldsymbol{D} \equiv\left[\begin{array}{c}
D_{1} \\
\vdots \\
D_{|\mathcal{F}|}
\end{array}\right]
$$

Notice that each block $A_{f f^{\prime}}^{s}$ is a function of $s$ and $\pi$; so is each matrix $D_{f}$. Consider the matrix

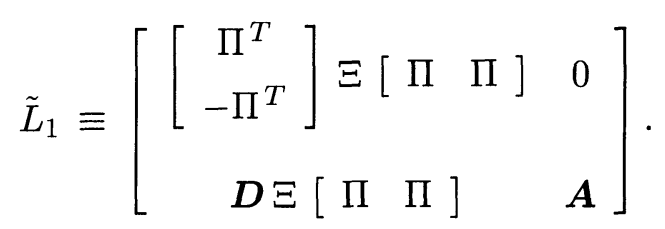

Clearly, $L_{1}$ is positive semidefinite if and only if $\tilde{L}_{1}$ is so. The next lemma shows that the latter matrix is positive semidefinite.

Lemma 1 For every compact set $\Omega \subset \Re^{(|\mathcal{F}| \times|\mathcal{N}|)+|\mathcal{N}|}$, there exists $\bar{\alpha}$ such that if

$$
\alpha_{i}<\bar{\alpha}, \quad \forall i \in \mathcal{N}
$$

the matrix $\tilde{L}_{1}$ is positive semidefinite for all $(s, \pi) \in \Omega$. 
Proof. The symmetric part of the matrix $\tilde{L}_{1}$ is equal to

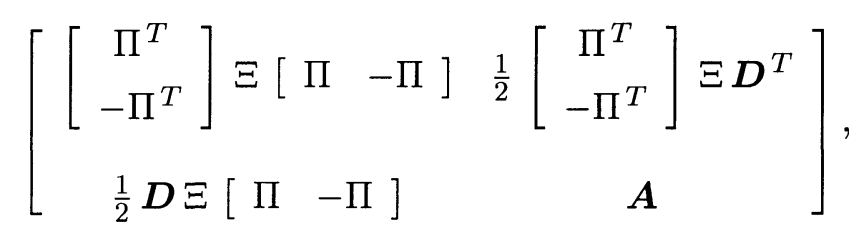

which we can write as the sum of two matrices:

$$
\left[\begin{array}{c}
\Pi^{T} \\
-\Pi^{T} \\
\frac{1}{2} \boldsymbol{D}
\end{array}\right] \Xi\left[\begin{array}{lll}
\Pi & -\Pi & \frac{1}{2} \boldsymbol{D}^{T}
\end{array}\right]+\left[\begin{array}{ccc}
0 & 0 & 0 \\
0 & 0 & 0 \\
0 & 0 & \boldsymbol{A}-\frac{1}{4} \boldsymbol{D} \Xi \boldsymbol{D}^{T}
\end{array}\right] .
$$

The first summand is clearly positive semidefinite. Provided that the pair $(s, \pi)$ is bounded, the matrix $\boldsymbol{A}-\frac{1}{4} \boldsymbol{D} \Xi \boldsymbol{D}^{T}$ is positive definite for all $\alpha_{i}$ with $\left|\alpha_{i}\right|$ sufficiently large.

Q.E.D.

Each pair $(\boldsymbol{s}, \boldsymbol{g})$ in the compact set $Y$ induces a price vector $\pi$ via the expression (9), where $\lambda^{ \pm}$is a solution of the LCP (14). The induced prices are bounded by the continuity of the function $\Phi$ and the boundedness of $Y$; cf. (17). Let $\Omega$ be a compact convex subset of $\Re(|\mathcal{F}| \times|\mathcal{N}|)+|\mathcal{N}|$ containing all such pairs $(s, \pi)$. Corresponding to this set $\Omega$, we may choose $\bar{\alpha}$ such that the Jacobian matrix of $\boldsymbol{F}_{\mathrm{II}}(\boldsymbol{x}, \varphi)$ is positive semidefinite for all pairs $(\boldsymbol{x}, \varphi)$ belonging to a convex set that contains all solutions of Model II. Thus $\boldsymbol{F}_{\text {II }}$ is monotone on this set. Based on this monotonicity property, we can establish the desired uniqueness of the sales and other variables in Model II.

Theorem 4 Under the assumptions of Theorem 2, there exists $\bar{\alpha}$ such that if

$$
\alpha_{i}<\bar{\alpha}, \quad \forall i \in \mathcal{N},
$$

the following variables are unique in the solutions of Model II:

(a) the sales $s_{f i}$ for all $f \in \mathcal{F}$ and $i \in \mathcal{N}$;

(b) the prices $\pi_{i}$ for all $i \in \mathcal{N}$;

(c) the total generations $\sum_{i \in \mathcal{N}} g_{f i}$ for all $f \in \mathcal{F}$; and

(d) the profits for each firm.

Proof. Let $\left(\boldsymbol{x}^{1}, \varphi^{1}\right)$ and $\left(\boldsymbol{x}^{2}, \varphi^{2}\right)$ be two solutions of Model II. Let $\pi^{1}$ and $\pi^{2}$ be the induced prices. By the monotonicity of $\boldsymbol{F}_{\text {II }}$, it follows that

$$
\left(\begin{array}{c}
\boldsymbol{x}^{1}-\boldsymbol{x}^{2} \\
\varphi^{1}-\varphi^{2}
\end{array}\right)^{T}\left(\boldsymbol{F}_{\mathrm{II}}\left(\boldsymbol{x}^{1}, \varphi^{1}\right)-\boldsymbol{F}_{\mathrm{II}}\left(\boldsymbol{x}^{2}, \varphi^{2}\right)\right)=0 .
$$


Let $\tilde{\lambda}^{i} \equiv \lambda^{+, i}-\lambda^{-, i}$ and $S^{i} \equiv \sum_{f \in \mathcal{F}} \sum_{i \in \mathcal{N}} s_{f i}$, for $i=1,2$. We have

$$
\begin{gathered}
\left(\tilde{\lambda}^{1}-\tilde{\lambda}^{2}\right)^{T} \Pi^{T} \Xi \Pi\left(\tilde{\lambda}^{1}-\tilde{\lambda}^{2}\right)+\omega\left(S^{1}-S^{2}\right)^{2}+ \\
\left(s^{1}-s^{2}\right)^{T}\left(h_{\mathrm{I}}\left(s^{1}, \lambda^{ \pm, 1}\right)-h_{\mathrm{I}}\left(s^{2}, \lambda^{ \pm, 2}\right)\right)=0 .
\end{gathered}
$$

By the mean-value theorem, it follows that for some triple $(\tilde{s}, \tilde{\pi})$ on the line segment joining $\left(s^{1}, \pi^{1}\right)$ and $\left(s^{2}, \pi^{2}\right)$,

$$
\begin{aligned}
& \left(\begin{array}{c}
\lambda^{+, 1}-\lambda^{+, 2} \\
\lambda^{-, 1}-\lambda^{-, 2} \\
\boldsymbol{s}^{1}-\boldsymbol{s}^{2}
\end{array}\right)^{T}\left[\begin{array}{c}
\Pi^{T} \\
-\Pi^{T}
\end{array}\right] \Xi\left[\begin{array}{ll}
\Pi & \Pi] \\
\boldsymbol{D} \Xi\left[\begin{array}{ll}
\Pi & \Pi
\end{array}\right] & \boldsymbol{A}
\end{array}\right]\left(\begin{array}{c}
\lambda^{+, 1}-\lambda^{+, 2} \\
\lambda^{-, 1}-\lambda^{-, 2} \\
\boldsymbol{s}^{1}-\boldsymbol{s}^{2}
\end{array}\right)+ \\
& \omega\left(S^{1}-S^{2}\right)^{2}=0,
\end{aligned}
$$

where the matrices $\boldsymbol{D}$ and $\boldsymbol{A}$ are evaluated at $(\tilde{\boldsymbol{s}}, \tilde{\pi})$. By the proof of Lemma 1, it follows that

$$
s^{1}=s^{2} \text { and } \Xi \Pi \tilde{\lambda}^{1}=\Xi \Pi \tilde{\lambda}^{2} .
$$

This yields $\pi^{1}=\pi^{2}$. Since

$$
\sum_{i \in \mathcal{N}} g_{f i}=\sum_{i \in \mathcal{N}} s_{f i}, \quad \forall f \in \mathcal{F},
$$

it follows that each firm's total generation is unique.

Finally, to show that the profit for each firm is unique, note that the profit of firm $f$ is equal to

$$
p_{f}(\boldsymbol{s}, \boldsymbol{\pi}, \boldsymbol{w}) \sum_{i \in \mathcal{N}} s_{f i}-\sum_{i \in \mathcal{N}}\left(c_{f i}-w_{i}\right) g_{f i}=\sum_{i \in \mathcal{N}}\left(\pi_{i}-c_{f i}\right) g_{f i},
$$

because $\pi_{i}=p_{f}(\boldsymbol{s}, \boldsymbol{\pi}, \boldsymbol{w})+w_{i}$ (by (6)) and the sum of $s_{f i}$ over all $i$ in $\mathcal{N}$ is equal to the sum of $g_{f i}$ over all $i$ in $\mathcal{N}$. Let $(\hat{\boldsymbol{x}}, \hat{\varphi})$ be an arbitrary solution of Model II. Consider the linear program in the variable $g_{f} \equiv$ $\left(g_{f i}: i \in \mathcal{N}\right)$ :

$$
\begin{array}{ll}
\text { maximize } & \sum_{i \in \mathcal{N}}\left(\hat{\pi}_{i}-c_{f i}\right) g_{f i} \\
\text { subject to } & 0 \leq g_{f i} \leq \mathrm{CAP}_{f i}, \quad \forall i \in \mathcal{N} \\
\text { and } & \sum_{i \in \mathcal{N}} g_{f i}=\sum_{i \in \mathcal{N}} \hat{s}_{f i} .
\end{array}
$$

Since $\hat{\pi}_{i}$ for $i \in \mathcal{N}$ and $\sum_{i \in \mathcal{N}} \hat{s}_{f i}$ are constants of Model II, it follows that the above linear program depends only on the firm $f$ and does not 
depend on the pair $(\hat{\boldsymbol{x}}, \hat{\varphi})$ of solution to Model II. The optimal objective value of the linear program gives the profit of firm $f$.

Q.E.D.

Acknowledgments. The work of this research was partially supported by the National Science Foundation under grant ECS-0080577.

\section{References}

[1] R. Baldick, R. Grant, and E. Kahn, "Linear Supply Function Equilibrium: Generalizations, Application, and Limitations," PWP-078, University of California Energy Institute, Berkeley, CA (August 2000).

[2] C.A. Berry, B.F. Hobbs, W.A. Meroney, R.P. O'Neill, and W.R. Stewart, Jr., "Analyzing Strategic Bidding Behavior in Transmission Networks," Utilities Policy 8 (1999) 139-158.

[3] R.W. Cottle, J.S. Pang, and R.E. Stone, The Linear Complementarity Problem, Academic Press (Boston 1992).

[4] C. Day, B.F. Hobbs, and J.S. Pang, "Oligopolistic Competition in Power Networks: A Conjectured Supply Function Approach," IEEE Transactions on Power Systems 17 (2002) 97-107.

[5] R. Gilbert and E. Kahn, editors, International Comparisons of Electricity Regulation, Cambridge University Press (New York 1996).

[6] F. Facchinei and J.S. Pang, Finite-Dimensional Variational Inequalities and Complementarity Problems, Springer-Verlag (New York 2003).

[7] B.F. Hobbs, "Linear Complementarity Models of Nash-Cournot Competition in Bilateral and POOLCO Power Markets," IEEE Transactions on Power Systems 16 (2001) 194-202.

[8] E. Kahn, "Numerical Techniques for Analyzing Market Power in Electricity," Electricity Journal 11 (July 1998) 34-43.

[9] P.D. Klemperer and M.A. Meyer, "Supply Function Equilibria," Econometrica 57 (1989) 1243-1277.

[10] Z.Q. Luo, J.S. Pang, and D. Ralph, Mathematical Programs with Equilibrium Constraints, Cambridge University Press (Cambridge 1996).

[11] C. Metzler, B.F. Hobbs, and J.S. Pang, "Nash-Cournot Equilibria in Power Markets on a Linearized DC Network with Arbitrage: Formulations and Properties", Network and Spatial Economics 3 (2003) 123-150.

[12] R.E. Schuler, "Analytic and Experimentally Derived Estimates of Market Power in Deregulated Electricity Systems: Policy Implications for the Management and Institutional Evolution of the Industry," Decision Support Systems 30 (January 2001) 341-355.

[13] F.C. Schweppe, M.C. Caramanis, R.E. Tabors, and R.E. Bohn, Spot Pricing of Electricity, Kluwer Academic Publishers, (Norwell 1988).

[14] Y. Smeers and J.Y. Wei, "Spatially Oligopolistic Model with Opportunity Cost Pricing for Transmission Capacity Reservations-A Variational Inequality Approach," CORE Discussion Paper 9717, Université Catholique de Louvain (February 1997). 